\title{
ON FAN'S EXTENSIONS OF BROWDER'S FIXED POINT THEOREMS FOR MULTI-VALUED INWARD MAPPINGS
}

\author{
CHENG-MING LEE and KOK-KEONG TAN \\ (Received 1 December 1976; revised 30 July 1977) \\ Communicated by E. Strzelecki
}

\begin{abstract}
Some fixed point theorems are obtained for weekly inward mappings which extend or generalize those results by K. Fan, B. Halpern or S. Reich. Various formulations of inward and outward concepts are also briefly discussed.
\end{abstract}

Subject classification (Amer. Math. Soc. (MOS) 1970): 47 H 10, 54 H 25.

\section{Introduction}

Recently K. Fan has established the following interesting result.

Theorem A (Fan, 1969, Theorem 5, p. 237). Let $K$ be a non-empty compact convex subset of a real Hausdorff topological vector space $E$. Let $f, g$ be two upper demi-continuous mappings defined on $K$ such that for each $x \in K, f(x)$ and $g(x)$ are non-empty subsets of $E$. Suppose that the following condition holds:

(1) For each $x \in K$, there exist three points $y \in K, u \in f(x)$ and $v \in g(x)$ such that $u-v=\lambda(y-x)$ for some $\lambda \in(0, \infty)$.

Then there exists a point $x_{0} \in K$ such that $f\left(x_{0}\right)$ and $g\left(x_{0}\right)$ cannot be strictly separated by a closed hyperplane.

We recall that a mapping $f$ from a topological space $X$ into the class of all non-empty subsets of a real Hausdorff topological vector space $E$ is upper demicontinuous on $X$ if for each $x \in X$ and for any open half-space $H$ in $E$ (that is, $H=\{u \in E: \varphi(u)<r\}$ for some continuous linear functional $\varphi$ on $E$, not identically zero, and for some real number $r$ ) containing $f(x)$, there exists a neighborhood $N$ of $x$ such that $f(y) \subset H$ for all $y \in N$. We also recall that two sets $A$ and $B$ contained in $E$ can be strictly separated by a closed hyperplane if and only if there exists a continuous linear functional $\varphi$ on $E$ and a real number $r$ such that $\varphi(x)>r$ for all $x \in A$ and $\varphi(y)<r$ for all $y \in B$.

The second author is partially supported by National Research Council of Canada under grant A-8096. 
Let $E$ be a real Hausdorff topological vector space, and $K$ a non-empty convex subset of $E$. Let

$\delta(K)=\{y \in K$ : there exists a point $z \in K$ such that $y+\lambda z \notin K$ for all $\lambda>0\}$, and for each $x \in K$,

$$
\begin{aligned}
I_{K}(x) & =\{x+\lambda(y-x): y \in K, \lambda>0\}, \\
O_{K}(x) & =\{x+\lambda(y-x): y \in K, \lambda<0\} .
\end{aligned}
$$

The set $\delta(K)$ is called the algebraic boundary of $K$ (Fan, 1969, p. 236) while the sets $I_{K}(x)$ and $O_{K}(x)$ are the inward set and outward set of $x$ with respect to $K$, respectively (Halpern, 1970, p. 88).

If $f: K \rightarrow E$ is such that for each $x \in K, f(x)$ is a non-empty closed convex subset of $E$, then $f$ is said to be weakly inward on $K$ (respectively weakly outward on $K$ ) if for each $x \in K, f(x) \cap \operatorname{cl}\left(I_{K}(x)\right) \neq \varnothing$ (respectively, $\left.f(x) \cap \operatorname{cl}\left(O_{K}(x)\right) \neq \varnothing\right)$.

Generalizing Browder's fixed point theorem (Browder, 1968, Theorem 2, p. 286), Halpern has proved the following result for weakly inward mappings:

THEOREM B (Halpern, $1^{\wedge} 70$, Theorem 2, p. 88). Let $K$ be a non-empty compact convex subset of a real locally convex Hausdorff topological vector space $E$ and $f$ be an upper semi-continuous mapping on $K$ which is weakly inward on $K$. Then there exists a point $x_{0} \in K$ such that $x_{0} \in f\left(x_{0}\right)$.

In order to extend Halpern's Theorem B from upper semi-continuous mappings to upper demi-continuous mappings (Reich, 1972, Corollary 2.2, p. 23), Reich used the following result:

THEOREM C (Reich, 1972, Proposition 2.1, p. 22). Let $K$ be a non-empty compact convex set in a real Hausdorff topological vector space $E$. Let $f, g$ be two upper demi-continuous functions on $K$ such that for each $x \in K, f(x)$ is a non-empty subset of $E$ and $g(x)$ is a non-empty compact subset of $E$. If

$$
f(x) \cap \mathrm{cl}\left(I_{K}(g(x)) \neq \varnothing \text { for every } x \in K,\right.
$$

then there exists a point $x_{0} \in K$ for which $f\left(x_{0}\right)$ and $g\left(x_{0}\right)$ cannot be strictly separated by a closed hyperplane.

The above result is a "weakly inward" version of Fan's Theorem A. It is, however, only a partial extension of Theorem A. In this paper, we prove Theorem 1, a "weakly inward" version of Theorem A which includes Theorem A and which also improves Theorem C. Finally, we use Theorem 1 to obtain results, unifying and generalizing Halpern's Theorem B for weakly inward mappings and Theorem 3 (Halpern, 1970, p. 89), for weakly outward mappings. Remarks concerning various formulations of inward and outward concepts are also briefly given. 


\section{Main results and applications}

The following result can be proved by applying the same argument in the first part in the proof of Theorem 5 (Fan, 1969, p. 237). Consequently its proof is omitted.

Lemma 1. Let $X$ be a compact Hausdorff space and let $E$ be a real Hausdorff topological vector space. Let $f$ and $g$ be two upper demi-continuous mappings defined on $X$ such that for each $X \in X, f(x)$ and $g(x)$ are non-empty subsets of $E$ that can be strictly separated by a closed hyperplane. Then there exists a mapping $\Psi$ of $X$ into $E^{\prime}$ (the dual of $E$ ) of the form $\Psi(x)=\sum_{i=1}^{n} a_{i}(x) \varphi_{i}$, where $a_{1}(x), \ldots, a_{n}(x)$ are nonnegative real-valued continuous functions on $X$ with $\sum_{i=1}^{n} a_{i}(x)=1$ for all $x \in X$ and $\varphi_{1}, \ldots, \varphi_{n} \in E^{\prime}$, such that for each $x \in X,[\Psi(x)](u)<[\Psi(x)](v)$, for all $u \in f(x)$ and $v \in g(x)$.

We now show that Theorem A remains true if the condition (1) is replaced by the weaker condition $(1)_{w}$.

THEOREM 1. Let $X$ be a non-empty compact convex subset of a real Hausdorff topological vector space $E$. Let $f$ and $g$ be two upper demi-continuous mappings defined on $K$ such that for each $x \in X, f(x)$ and $g(x)$ are non-empty subsets of $E$. Suppose that the following condition holds:

(1) For each $x \in X$, there exist two points $u \in f(x)$ and $v \in g(x)$ and two nets $u_{\alpha}, v_{\alpha}$ in $E$ and $a$ net $y_{\alpha}$ in $X$ such that $u_{\alpha} \rightarrow u, v_{\alpha} \rightarrow v$ and $u_{\alpha}-v_{\alpha}=\lambda_{\alpha}\left(y_{\alpha}-x\right)$ for some net $\lambda_{\alpha}$ in $(0, \infty)$.

Then there exists a point $x_{0} \in X$ such that $f\left(x_{0}\right)$ and $g\left(x_{0}\right)$ cannot be strictly separated by a closed hyperplane.

Proof. Suppose that the conclusion of the theorem is false. Then by Lemma 1, there exists a mapping $\Psi$ on $X$ into $E^{\prime}$ of the form $\Psi(x)=\sum_{i=1}^{n} a_{i}(x) \varphi_{i}$, where $a_{1}(x), \ldots, a_{n}(x)$ are non-negative real-valued continuous functions on $X$ with $\sum_{i=1}^{n} a_{i}(x)=1$ for all $x \in X$ and $\Psi_{1}, \ldots, \Psi_{n} \in E^{\prime}$, such that for each $x \in X$,

$$
[\Psi(x)](u)<[\Psi(x)](v) \text {, for all } u \in f(x) \text { and } v \in g(x) \text {. }
$$

Since $[\Psi(x)](y-x)=\sum_{i=1}^{n} a_{i}(x) \Psi_{i}(y-x)$ is a continuous mapping of $(x, y)$ on $X \times X$, the set $A=\{(x, y) \in X \times X:[\Psi(x)(y-x) \geqslant 0\}$ is closed in $X \times X$. Clearly, for each $x \in X,(x, x) \in A$ and the set $\{y \in X:(x, y) \notin A\}$ is convex or empty. Thus by Lemma 4 (Fan, 1961, p. 309) there exists a point $x_{0} \in X$ such that $\left\{x_{0}\right\} \times X \subset A$. Thus

$$
\left[\Psi\left(x_{0}\right)\right]\left(y-x_{0}\right) \geqslant 0 \text { for all } y \in X \text {. }
$$

Let $u, v, u_{\alpha}, v_{\alpha}, y_{\alpha}$ and $\lambda_{\alpha}$ be given as in (1) for $x=x_{0}$. Since $\Psi\left(x_{0}\right)$ is linear and $\lambda_{\alpha}>0$, from (2) we see that

$$
\left[\Psi\left(x_{0}\right)\right]\left(u_{\alpha}-v_{\alpha}\right)=\lambda_{\alpha}\left[\Psi\left(x_{0}\right)\right]\left(y_{\alpha}-x_{0}\right) \geqslant 0 .
$$


Since $\Psi\left(x_{0}\right)$ is continuous and $u_{\alpha} \rightarrow u$ and $v_{\alpha} \rightarrow v$, we have $\left[\Psi\left(x_{0}\right)\right](u-v) \geqslant 0$, which contradicts (1). This proves the theorem.

With slight modification in the proof of the above theorem, we have the following result:

THEOREM 2. Let $X$ be a non-empty compact convex subset of a Hausdorff topological vector space, and let $E$ be a real Hausdorff topological vector space. Suppose that $G$ is a continuous convex mapping from $X$ into $E$ and that $F$ is an upper demicontinuous mapping defined on $X$ such that for each $x \in X, F(x)$ is a non-empty subset of $E$. Suppose that the following condition holds:

(2) For each $x \in X$, there exists a point $u \in F(x)$, a net $y_{\alpha}$ in $X$, and a net $u_{\alpha}$ in $E$ such that $u_{\alpha} \rightarrow u$ and $u_{\alpha}-G(x)=\lambda_{\alpha}\left(G\left(y_{\alpha}\right)-G(x)\right)$ for some net $\lambda_{\alpha}$ in $(0, \infty)$. Then there exists a point $x_{0} \in X$ such that $G\left(x_{0}\right)$ and $F\left(x_{0}\right)$ cannot be strictly separated by a closed hyperplane.

We shall now apply the theorems to obtain some fixed point theorems.

THEOREM 3. Let $K$ be a non-empty compact convex subset of a real Hausdorff topological vector space $E$. Let $f, g$ be two demi-continuous mappings defined on $K$ such that for each $x \in K, f(x)$ and $g(x)$ are non-empty subsets of $E$. Suppose that the following conditions hold:

(3) For each $x \in \delta(K)$, there exists a point $u \in f(x)$, a net $u_{\alpha}$ in $E$ and a net $z_{\alpha}^{1}$ in $K$ such that $u_{\alpha} \rightarrow u$ and $u_{\alpha}-x=\beta_{\alpha}\left(z_{\alpha}^{1}-x\right)$ for some net $\beta_{\alpha}$ in $(0, \infty)$.

(4) For each $x \in \delta(K)$, there exists a point $v \in g(x)$, a net $v_{\alpha}$ in $E$ and a net $z_{\alpha}^{2}$ in $K$ such that $v_{\alpha} \rightarrow v$ and $v_{\alpha}-x=\gamma_{\alpha}\left(z_{\alpha}^{2}-x\right)$ for some net $\gamma_{\alpha}$ in $(-\infty, 0)$.

Then there exists a point $x_{0} \in K$ such that $f\left(x_{0}\right)$ and $g\left(x_{0}\right)$ can not be strictly separated by a closed hyperplane.

REMARK 1. Similar to the inward case pointed out by Fan (1969, p. 236), it is easily checked that the condition (3) holds if and only if $f$ is weakly inward on $K$ and the condition (4) holds if and only if $g$ is weakly outward on $K$. Also, the condition $(1)_{\mathrm{w}}$ is equivalent to the condition obtained by replacing " $x \in X$ " by " $x \in \delta(X)$ " in the condition $(1)_{\mathrm{w}}$.

Proof of Theorem 3. (This is the same proof as in Theorem 8, Fan (1969), p. 239.) For each $x \in \delta(K)$, let $u, u_{\alpha}, v, v_{\alpha}$ et cetera be as given in (3) and (4), and then let

$$
y_{\alpha}=\frac{\beta_{\alpha}}{\beta_{\alpha}-\gamma_{\alpha}} z_{\alpha}^{1}+\frac{-\gamma_{\alpha}}{\beta_{\alpha}-\gamma_{\alpha}} z_{\alpha}^{2}, \quad \lambda_{\alpha}=\beta_{\alpha}-\gamma_{\alpha} .
$$

Then one sees that the condition (1) $)_{\mathrm{w}}$ is satisfied for all $x \in \delta(K)$, and hence by Remark 1 , the assertion of Theorem 3 follows from Theorem 1 . 
REMARK 2. We will say that the mapping $f$ is weakly inward with respect to $g$ on $K$ provided that $(1)_{\mathrm{w}}$ is satisfied. Then from the proof of Theorem 3 , one sees that if $f$ is weakly inward on $K$ and $g$ is weakly outward on $K$, then $f$ is weakly inward with respect to $g$ on $K$.

THEOREM 4. Let $K$ be a non-empty compact convex subset of a real locally convex Hausdorff topological vector space E. Let $f, g$ be two upper demi-continuous mappings defined on $K$ such that for each $x \in K, f(x)$ and $g(x)$ are closed and convex, and at least one of them is compact. If $f$ is weakly inward and $g$ is weakly outward on $K$, or more generally if $f$ is weakly inward with respect to $g$ on $K$, then there exists $a$ point $x_{0} \in K$ such that $f\left(x_{0}\right) \cap g\left(x_{0}\right) \neq \varnothing$.

Proof. This follows from Theorem 1 and Theorem 3 and the fact that in the space concerned, a closed convex set and a compact convex set without common points can be strictly separated by a closed hyperplane.

Remark 3. Taking either $f$ or $g$ in Theorem 4 to be the identity mapping on $K$, one obtains results generalizing Halpern's fixed point Theorems 2 and 3 (Halpern, 1970 , pp. 88, 89).

ReMARK 4. Let

$$
I_{K}(g(x))=\{v+\lambda(y-x): v \in g(x), y \in K, \lambda>0\} .
$$

Then one sees that the Fan's condition (1) holds if and only if the following condition holds:

(5) For each $x \in K, f(x) \cap I_{K}(g(x)) \neq \varnothing$.

However, the condition (1) is not equivalent to the condition:

$(5)_{\mathrm{w}}$ For each $x \in K, f(x) \cap \mathrm{cl} I_{K}(g(x)) \neq \varnothing$.

It is not known whether all the other conditions imposed on $f, g, K$ and $E$ in Theorem 1 force the conditions (1) and (5) $)_{\mathrm{w}}$ to be equivalent. However, the following partial solution is immediate.

Proposition. Let $K$ be a convex subset of $E$ and $f, g$ be mappings from $K$ into the class of all non-empty subsets of $E$ and suppose that $g(x)$ is compact for each $x \in K$. Then the conditions $(1)_{\mathrm{w}}$ and $(5)_{\mathrm{w}}$ are equivalent.

Remark 5. By the above proposition, we see that Theorem $\mathrm{C}$ is a corollary of Theorem 1 here. Note that Theorem 1 is a true generalization of Theorem $A$, while Theorem $\mathrm{C}$ is not. 
Remark 6. Theorem 5 below is a direct consequence of Theorem 2. Noting that both Theorems 2 and 5 also hold for " $\lambda_{\alpha}$ in $(-\infty, 0)$ " instead of " $\lambda_{\alpha}$ in $(0,+\infty)$ ", one sees easily that Theorem 5 also generalized both Halpern's fixed point theorems.

THEOREM 5. In addition to the assumption in Theorem 2 , let $E$ be locally convex and for each $x \in X, F(x)$ be closed and convex. Then there exists $x_{0} \in X$ such that $G\left(x_{0}\right) \in F\left(x_{0}\right)$.

REMARK 7. As another application of Theorem 2, we give the following result, which is a variant of Theorem 4.

THEOREM 6. Let $K$ be a non-empty compact convex subset of a real locally convex Hausdorff topological vector space $E$. Let $f, g$ be two mappings defined on $K$ such that for each $x \in K, f(x)$ and $g(x)$ are non-empty closed convex subjects of $E$. Let I be the identity mapping on $K$. Suppose that the mapping $I+f-g$ is upper demi-continuous and weakly inward on $K$. Then there exists $x_{0} \in K$ such that $f\left(x_{0}\right) \cap g\left(x_{0}\right) \neq \varnothing$.

Proof. Denote $K=X$, and write $F=I+f-g$ and $G=I$. Then one shows that all the assumptions in Theorem 2 are satisfied. Hence, there exists $x_{0} \in K$ such that $x_{0}$ and $F\left(x_{0}\right)=x_{0}+f\left(x_{0}\right)-g\left(x_{0}\right)$ cannot be strictly separated by a closed hyperplane. As $x_{0}+f\left(x_{0}\right)-g\left(x_{0}\right)$ is closed and convex, one concludes that

$$
x_{0} \in x_{0}+f\left(x_{0}\right)-g\left(x_{0}\right) \text {, so that } f\left(x_{0}\right) \cap g\left(x_{0}\right) \neq \varnothing \text {. }
$$

\section{References}

F. E. Browder (1968), “The fixed point theory of multi-valued mappings in topological vector spaces", Math. Ann. 177, 283-301.

K. Fan (1961), "A generalization of Tychonoff;s fixed point theorem", Math. Annalen 142, 305-310.

K. Fan (1969), "Extensions of two fixed point theorems of F. E. Browder", Math. Z. 112, 234-240.

B. Halpern (1970), "Fixed point theorems for set-valued maps in infinite dimensional spaces", Math. Ann. 189, 87-98.

S. Reich (1972), "Fixed points in locally convex spaces", Math. Z. 125, 17-31.

Department of Mathematics

University of Wisconsin, Milwaukee

Milwaukee, Wisconsin 53201

U.S.A.
Department of Mathematics

Dalhousie University

Halifax

Nova Scotia

Canada 